\title{
SAXS study on the crystallization of PET under physical confinement in PET/PC multilayered films
}

\author{
I. Puente Orench ${ }^{\mathrm{a}, *}$, N. Stribeck ${ }^{\mathrm{b}}, \mathrm{F}$. Ania ${ }^{\mathrm{a}}, \mathrm{E}$. Baer $^{\mathrm{c}}$, A. Hiltner ${ }^{\mathrm{c}}$, F.J. Baltá Calleja ${ }^{\mathrm{a}}$ \\ a Instituto de Estructura de la Materia, CSIC, Serrano 119, 28006 Madrid, Spain \\ ${ }^{\mathrm{b}}$ Institute of Technical and Macromolecular Chemistry, University of Hamburg, Bundesstr. 45, 20146 Hamburg, Germany \\ ${ }^{\mathrm{c}}$ Department of Macromolecular Science and Engineering, Center for Applied Polymer Research, Case Western Reserve University, Cleveland, OH, USA
}

\section{A R T I C L E I N F O}

\section{Article history:}

Received 7 December 2008

Received in revised form

24 March 2009

Accepted 26 March 2009

Available online 7 April 2009

\section{Keywords:}

Confinement

Crystallization

Multilayers

\begin{abstract}
A B S T R A C T
The present work is concerned with the study of the development of the crystalline structure of poly(ethylene terephthalate) in multilayered films of poly(ethylene terephthalate)/polycarbonate (PET/ PC) prepared by means of layer multiplying coextrusion. Small angle X-ray scattering patterns were recorded during isothermal crystallization experiments and evaluated by means of Ruland's interface distribution function. Thus, structural parameters describing the thickness distribution of crystalline and amorphous layers were determinated. It is shown that the crystallization of PET is delayed with increasing confinement. However when the crystallization process comes to an end, the values of the nanostructural parameters of the lamellar system are nearly the same for the confined and non-confined PET.
\end{abstract}

(C) 2009 Elsevier Ltd. All rights reserved.

\section{Introduction}

It is well known that a reduced dimensionality of materials may result in interesting phenomena. In particular, for polymer systems it has been found that confinement can change the glass transition temperature [1-4], molecular mobility [5,6], behaviour of the phases and morphology [7,8], molecular orientation [9] and crystallization behaviour [10-13]. Polymeric materials can be subjected either to chemical or to physical confinement. Whereas physical confinement is only restricting the volume of the material, chemical confinement is restricting as well, molecular motions by chemical bonds [14].

The crystallization of polymers in physically confined environments is a topic of increasing interest because of the required basic understanding of crystallization in the application of polymers for nanotechnologies. In studies of confined crystallization of polymers, very particular effects have been found. The mechanisms of nucleation are affected due to the reduced number of chain conformations and interacting molecules in comparison with the bulk [11]. Lamellar growth rate and the final degree of crystallinity are diminished if the chains in thin polymer films are

\footnotetext{
* Corresponding author. Present address: Spanish CRG's D1B \& D15 - Instituto de Ciencia de Materiales de Aragón CSIC - Institut Laue Langevin, 6 rue Jules Horowitz, 38042 Grenoble, France. Tel.: +33 476207477; fax: +33 476483906.

E-mail address: puenteorench@ill.eu (I. Puente Orench).
}

subjected to a preferred orientation $[10,15]$. Confined crystallization of polypropylene layers in between amorphous layers of polystyrene results in discoidal morphologies which transform into long stacks of very short lamellae arranged in fan-like arrays, when decreasing layer thickness from the microscale to the nanoscale [16]. The physical confinement of poly( $\varepsilon$-caprolactone) $(\mathrm{PCL})$ in blends with a lamellar morphology of poly( $\varepsilon$-caprolactone)/poly(styrene)- $b$-poly(ethylenepropylene) (PCL/PS-PEP) has shown to affect both the morphology and the orientation of PCL crystals [14].

Confined polymer layers are readily prepared by layer multiplying coextrusion technique, which can produce laminar architectures up to thousands of layers $[17,18]$. The thickness of the individual layers of these multilayered films can be tuned from the micrometer to the nanometer range, being proven that they are very suitable materials to study the effects of physical confinement on the structure formation of semi-crystalline polymers [16,19]. Two immiscible polymers, poly(ethylene terephthalate) (PET) and polycarbonate $(\mathrm{PC})$, have been used to fabricate the multilayered films. A previous investigation of the nanostructure of PET in the confined state under physical confinement, performed on annealed PET/PC multilayered films [20], has already shown that, with increasing confinement (i.e. when the PET layer becomes thinner), the volume crystallinity of the selectively crystallized PET layers decreases. Furthermore, confinement induces an increase of the long period (i.e. the distance between neighboring crystalline lamellae) of semi-crystalline PET. These effects are observed when 
the thickness of the PET layers becomes smaller than $1 \mu \mathrm{m}$. The crystallization temperature at which drastic changes between confined and non-confined PET were found is $117^{\circ} \mathrm{C}$.

The aim of the present study is to investigate the evolution of the nanostructure. For this purpose the real time small angle X-ray scattering (SAXS) method is used to monitor the isothermal crystallization of PET confined between amorphous layers of PC. For data analysis, the scattering patterns are transformed into Ruland's interface distribution function (IDF) [21]. The IDF, $g(r)$, represents the probability distribution of finding two interfaces between a crystalline and an amorphous lamella at a certain distance $r$. The IDF can be fitted by models which describe the sizes and the statistical arrangement of the lamellae in the material [22,23]. In this way the structural parameters are determined. Parameters of interest are the average thickness of crystals, $l_{c}$, the thickness of the amorphous region between crystals, $l_{\mathrm{a}}$, and the long period, $L$ (average distance between adjacent lamellar crystals). As far as we know this is the first time that structural parameters for polymer nanolayered systems are reported.

\section{Experimental section}

\subsection{Materials}

The materials used in this work are commercial thermoplastic polyesters: poly(ethylene terephthalate) (PET) (M\&G Cleartuf 8004) and polycarbonate (PC) (DOW Calibre 200-10). Both materials have a molecular weight of 30000 . PET/PC multilayered films with 1024 layers, total thickness of $\sim 75 \mu \mathrm{m}$ and PET/PC volume ratios of $100 /$ 0 (pure PET), 70/30, 50/50, and 30/70 were fabricated by means of layer multiplication coextrusion technique described elsewhere [22]. The thicknesses of the individual PET layers are $70 \mathrm{~nm}$ for the coextruded PET (100/0) film, and 100, 70 and $50 \mathrm{~nm}$ for the $70 / 30$, 50/50 and 30/70 PET/PC coextruded multilayered films respectively. As a matter of fact, the coextruded pure PET film loses the layered structure during processing and finally behaves as bulk PET. The asprocessed multilayered films were in the glassy-amorphous state. The PET layers of the composite can be crystallized selectively by thermal treatments, while the PC layers remain in the amorphous state, because the glass transition temperature of these polymers is quite different $\left(75^{\circ} \mathrm{C}\right.$ for PET and $150^{\circ} \mathrm{C}$ for PC). Thus, the changes occurring in the nanostructure of PET during crystallization in a physically confined environment have been studied.

\subsection{SAXS measurements}

Time-resolved SAXS experiments during isothermal crystallization at $117^{\circ} \mathrm{C}$ were performed at the beam line $\mathrm{A} 2$ at HASYLAB, Hamburg (Germany) using synchrotron radiation of $0.15 \mathrm{~nm}$. The multilayered films were mounted in the furnace of the beam line A2 [24] in normal transmission geometry, i.e. the film surface normal was parallel to the X-ray beam. As we knew already from a study [20] in this geometry the recorded SAXS patterns always look as if the material were isotropic. The scattering patterns of the machine background and of the samples were exposed for $120 \mathrm{~s}$ and recorded on a two-dimensional MAR-CCD-165 detector placed at a distance of $2050 \mathrm{~mm}$ from the sample. The distance was verified by means of the scattering from a rat-tail tendon standard. The flux of the primary beam was measured before and after penetrating the sample.

\subsection{SAXS analysis}

All two-dimensional scattering images were normalized for detector efficiency, incident beam flux, and exposure time. The machine background was multiplied by the sample absorption and subtracted. The first pattern of each series taken at $117^{\circ} \mathrm{C}$ still showed the diffuse scattering of an amorphous sample $\left(I_{0}\right)$. It was used as an amorphous background and subtracted from each of the following patterns. Thus, a pre-evaluated scattering intensity of the semi-crystalline nanostructure, $[I(\mathbf{s})]_{2}\left(s_{1}, s_{3}\right)$ : $=\lceil I]_{2}\left(s_{1}, s_{3}\right)$, was obtained. Here $\boldsymbol{s}=\left(s_{1}, s_{2}, s_{3}\right)$ is the scattering vector with its modulus $s=|\boldsymbol{s}|=(2 / \lambda) \sin \theta, \lambda$ is the X-ray wavelength and $2 \theta$ is the scattering angle. Because the X-ray beam is parallel to the normal surface of the multilayered films $\left(s_{2}\right.$ direction) not the complete scattering $I(\boldsymbol{s})$ in reciprocal space is measured, but only the intensity $[I]_{2}\left(s_{1}, s_{3}\right)$ in the $\left(s_{1}, s_{3}\right)$-plane.

As it has been shown in a previous work [20], the irradiation in $s_{1}$ direction (i.e. parallel to the film surface), $[I]_{2}\left(s_{2}, s_{3}\right)$ generates completely different scattering patterns showing a strong equatorial streak from the multilayer structure and an anisotropic scattering, when PET is crystallized in the thinner layers. Therefore, it is impossible to record the complete scattering $I(\mathbf{s})$ in the timeresolved experiment and we have to resort to the nanostructure information contained in $[I]_{2}\left(s_{1}, s_{3}\right)$. According to scattering theory [23] the two-dimensional Fourier transformation, $\mathfrak{\Im}_{2}()$, of the measured intensity is related to the two-dimensional projection, \{\}$_{2}$, of the correlation function $P(r)$ in real space $\{P\}_{2}\left(r_{1}, r_{2}\right)=$ $\mathfrak{J}_{2}\left([I]_{2}\left(s_{1}, s_{3}\right)\right)$. Ultimately, our analysis remains limited to variations of this projected nanostructure information.

The measured intensities $[I]_{2}\left(s_{1}, s_{3}\right)=[I]_{2}\left(s_{13}, \phi\right)=[I]_{2}\left(s_{13}\right)$ were always found to be isotropic in the measured $\left(s_{1}, s_{3}\right)$-plane. Here $s_{13}=\left(s_{1}^{2}+s_{3}^{2}\right)^{0.5}$ and $\phi=\arctan \left(s_{3} / s_{1}\right)$ is the azimuthal angle. Thus, taking advantage of the available two-dimensional data, we carried out azimuthal averaging $[I]_{2}\left(s_{13}\right)=1 /\left(2 \pi s_{13}\right)$ $\int_{0}^{2 \pi}[I]_{2}\left(s_{13}, \phi\right) \mathrm{d} \phi$ in order to increase the signal-to-noise ratio in the resulting scattering curve.

We are assuming that the emerging scattering is related to the semi-crystalline nanostructure of a lamellar system. In such a system the scattering of each layer stack is described by a onedimensional intensity $I_{1}\left(s_{x}\right)$, with $s_{x}$ the direction normal to the lamellae. If the orientation distribution $s_{x}$ of the layer stacks were completely at random, we would observe an isotropic scattering, $I(s)$, and the representative one-dimensional scattering is obtained by solid-angle integration, which is simplified as:

$I_{1}(x)=2 \pi s^{2} I(s)$

for isotropic scattering in three-dimensional space. Equation (1) is called Lorentz correction [23]. Although our data are only isotropic in two dimensions, we followed the usual method, carried out a Lorentz correction $\tilde{I}\left(s_{13}\right)=s_{13}^{2}[I]_{2}\left(s_{13}\right)$ and determined a long period $L=1 / s_{13 m}$ from $s_{13 m}$, the position of the peak maximum in this curve. Bearing in mind the above-mentioned relations we will, in general, use a simplified notation, e.g. $I(s)$ instead of $[I]_{2}\left(s_{13}\right)$.

From $I(s)$ an interface distribution function [21] $g(r)$ was computed considering $I(s)$ as a one-dimensional section in reciprocal space that was measured by a point focus. The evaluation of these one-dimensional curves was carried out by the program TOPAS [25]. The first step was the determination of the deviations [26] from the ideal Porod's law. For this purpose $\ln \left(s^{4} I(s)\right)$ was plotted vs. $s^{2}$ [23]. From slope and intercept of the Porod-line the parameters $A_{\mathrm{P}}$ (Porod asymptote) and $d_{\mathrm{z}}$ (width of the transition zone at the interface of the crystalline lamella between crystalline and amorphous density) were determined. With these parameters the interference function of ideal lamellar two-phase system $G(s)=s^{4} I(s) \exp \left[\left(4 / 9 \pi^{2} d_{z}^{2} s^{2}\right)\right]-A_{P}$ was computed. The onedimensional Fourier transform of $G(s)$ is the interface distribution function 
$g(r)=\mathfrak{J}(G(s))$

We should keep in mind that in our special case this function does not necessarily describe the nanostructure of the lamellar stacks, but is only an approximation of their projection on the $r_{13}-$ plane of the multilayered film.

The IDFs were analyzed by model fitting using the SIMPLEX [27] algorithm. The data were best fitted by the program mr_stap [25] which implements a (paracrystalline) stacking model [28]. Resulting parameters are the average layer thicknesses of the two phases, $l_{1}$ and $l_{2}$, as well as the relative standard deviations $\sigma_{1} / l_{1}$ and $\sigma_{2} / l_{2}$ of the layer thickness distributions.

\section{Results and discussion}

Fig. 1 illustrates the Lorentz-corrected SAXS curves recorded during the isothermal crystallization at $117^{\circ} \mathrm{C}$ as a function of crystallization time $t_{\mathrm{c}}$ and the scattering vector $s$ for the pure PET and the PET/PC multilayered films with 100, 70 and $50 \mathrm{~nm}$ of PET layer thickness. With increasing confinement, it is clearly observed a delay in the first appearance of the SAXS maximum. Moreover, before the occurrence of a real maximum, one observes an increase of scattering intensity due to the formation of non-spatially correlated crystalline entities. This period of time it is known as induction period. A commonly accepted view to describe the initial stages of crystallization in polymers is still missing. Different approaches including the classical nucleation and growth theory, the stepwise formation of lamellae by merging of mesomorphic domains or the spinodal decomposition type microphase separation have been proposed [29]. The induction period is the time prior to the formation of stable crystal nuclei. Fig. 2 represents the evolution in time of the intensity at the SAXS maximum. It is observed that coextruded pure PET exhibits an induction period of around $4 \mathrm{~min}$ while the PET/PC film whose PET layers are $50 \mathrm{~nm}$ thick needs 20 min to display a clear SAXS maximum. During the crystallization of PET layers, this maximum shifts towards higher $s$ values. Assuming that this scattering comes from the electron density variation among crystalline and amorphous regions within the PET layers, this indicates that confinement of PET delays the formation of the semi-crystalline lamellar stacks.

From the position of the maximum, the long period, $L$ has been calculated. Fig. 3 shows $L$ as a function of the crystallization time $t_{\mathrm{c}}$. The long period, $L$, provides information about the average distance between correlated structures with similar electron density. As crystallization progresses $L$ decreases. This can be explained invoking two non exclusive structural models: 1 ) the formation of new crystals in the inter-lamellar amorphous phase and 2) the formation of new stacks of crystals with shorter correlation length within the amorphous interstack regions.

As $L$ decreases, it is possible to detect two steps during the crystallization process. First a period of time during which $L$ decreases at a higher rate, probably due to the formation of new crystals and a second period where $L$ decreases slowly, which could be related to the perfecting or thickening of the former crystals and occasionally with the occurrence of new crystals.

In a two-phase system, the SAXS intensity arises from the difference in average electron density between the phases [30,31]. The total integrated intensity of the SAXS pattern (in three
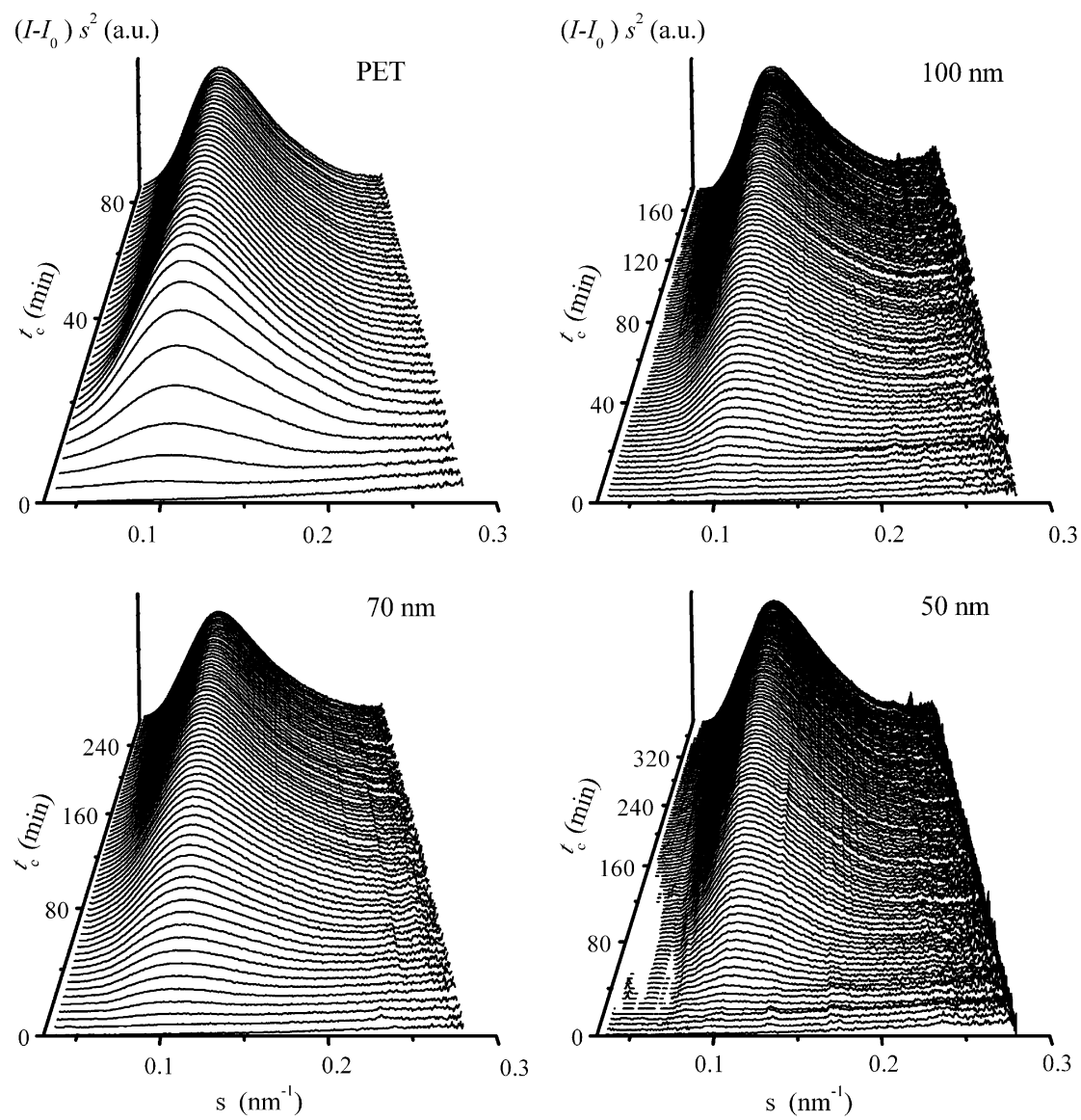

Fig. 1. Evolution of SAXS maximum during isothermal crystallization at $117^{\circ} \mathrm{C}$ for PET (100/0), PET/PC: $100 \mathrm{~nm}(70 / 30), 70 \mathrm{~nm}(50 / 50)$ and $50 \mathrm{~nm}(30 / 70)$. 


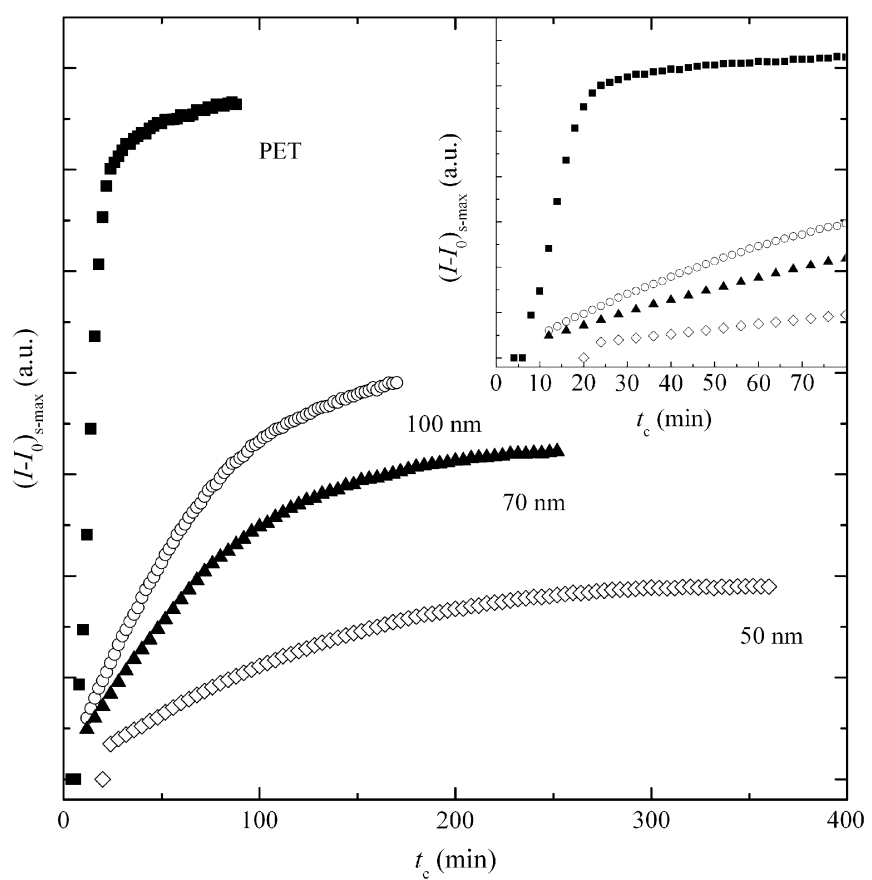

Fig. 2. Intensity at SAXS maximum $\left(I-I_{0}\right)_{s-\max }$, during isothermal crystallization at $117^{\circ} \mathrm{C}$ vs. crystallization time $t_{\mathrm{c}}$.

dimensions) is an invariant quantity, $Q$. In the case of an isotropic two-phase system, $Q$ is proportional to the product of the volume fraction and difference in electron density of the phases

$Q=4 \pi \int I(s) s^{2} \mathrm{~d} s=\varphi_{1} \varphi_{2}\left(\rho_{1}-\rho_{2}\right)^{2}$

where $\varphi_{1}$ and $\varphi_{2}$ are the volume fractions of the phases and $\rho_{1}$ and $\rho_{2}$ their respective electron densities. Although the scattering

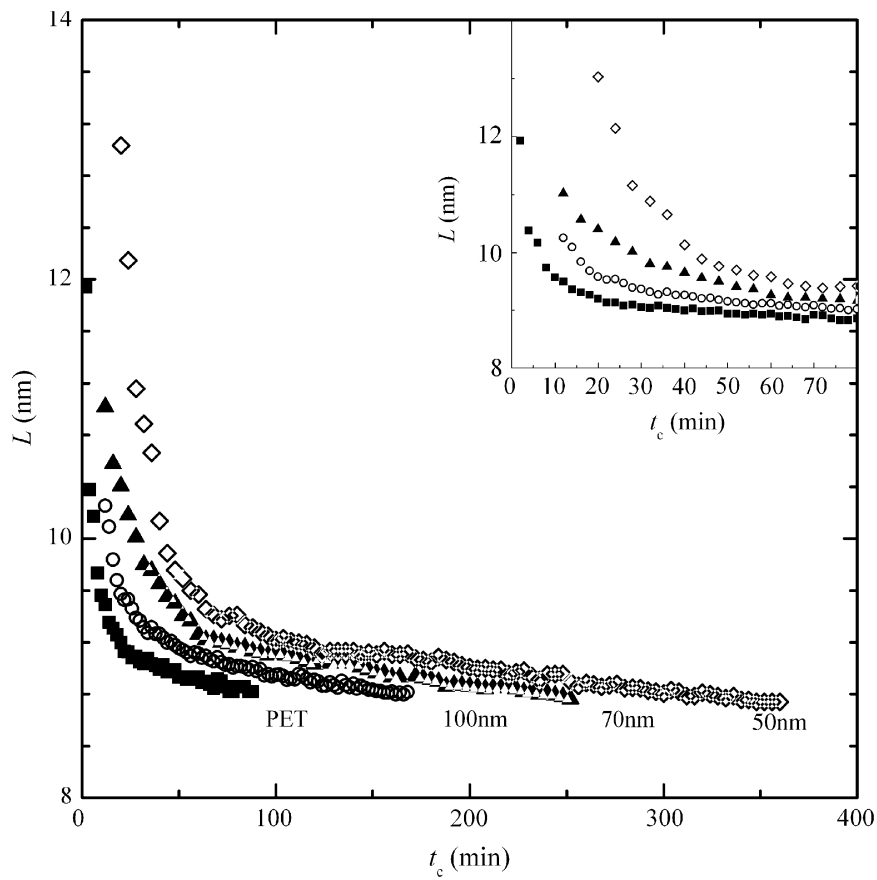

Fig. 3. Long period, $L$, computed from Bragg's law during isothermal crystallization at $11{ }^{\circ} \mathrm{C}$ vs. crystallization time $t_{\mathrm{c}}$. patterns of the PET/PC multilayered films are only isotropic in normal transmission geometry, we have followed the classical method for computing the invariant.

Fig. 4 illustrates the normalized invariant $Q-Q_{0}$, as a function of crystallization time (first amorphous frame at $117{ }^{\circ} \mathrm{C}$ has been subtracted and normalization by PET layer thickness has been performed). Taking for granted that the difference in electron density between the crystalline and amorphous phase of PET does not change during crystallization, the invariant can be related to the product of the crystalline and amorphous volume fractions in the PET layers. The two steps in the crystallization process can be also recognized, in the time variation of $Q-Q_{0}$ : firstly a rapid increase at a constant rate (primary crystallization) and thereafter, a much lower rate of increase. The final $Q-Q_{0}$ values should be proportional to the total degree of crystallinity of PET. Although the $Q$ data for the $100 \mathrm{~nm}$ and $70 \mathrm{~nm}$ thick samples did not reach an asymptotic value, the obtained results suggest that confinement reduces the degree of crystallinity of the studied multilayered materials. In a previous paper [20], the measured volume degree of crystallinity of PET at room temperature after annealing at $150^{\circ} \mathrm{C}$ of a sample of coextruded pure PET and that of a multilayered PET/PC film with a PET layer thickness of $50 \mathrm{~nm}$ was $36 \%$ and $32 \%$ respectively, a relative decrease of more than $10 \%$. Two possible explanations have been tentatively proposed to account for this decrease. The first one is related to interactions between the polymer and the interfaces. The crystallization of ultrathin films with strong interactions between the polymer and the substrate on which the film was prepared has been shown to yield the reduction of the mobility in proximity to the interface. Such regions would influence the dynamics of the whole polymer film, when the dimension of the sample is comparable with the length scale of the interfacial interaction. The second explanation accounts for the reduction of the number of active nuclei taking part in the conversion of the amorphous phase into crystals. The probability to find a nucleus in a thin layer should decrease with the reduction of the thickness, tending to zero for the thinnest films [32].

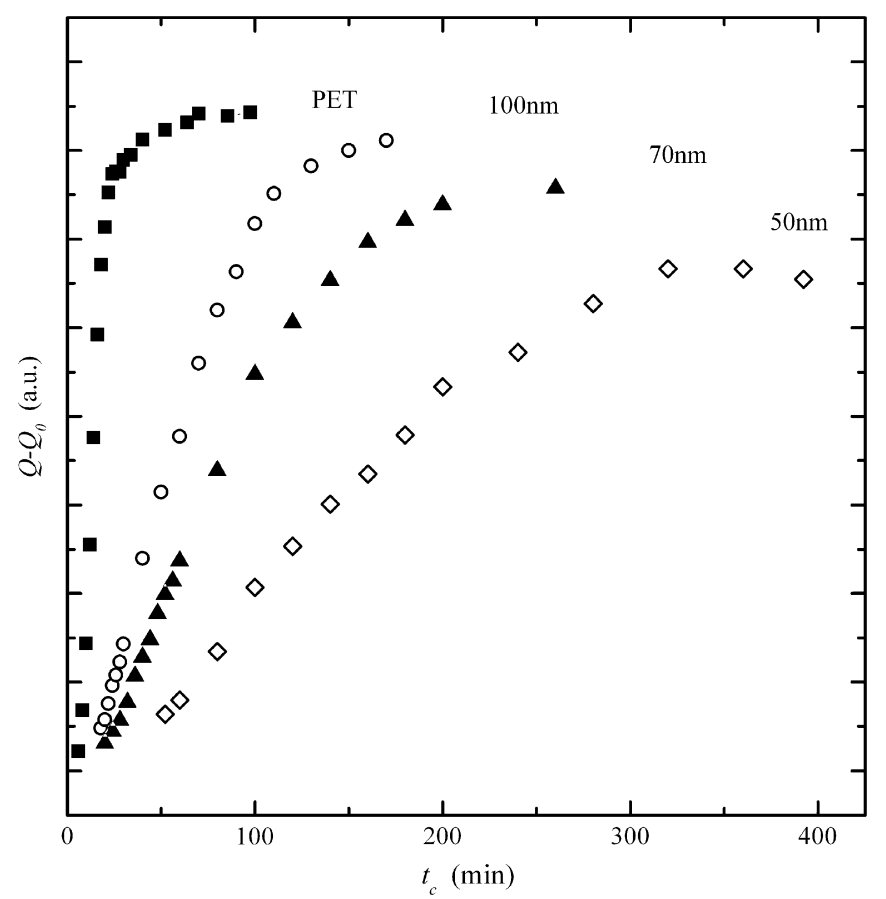

Fig. 4. Variation of the invariant $Q-Q_{0}$ during isothermal crystallization at $117^{\circ} \mathrm{C}$. 
Fig. 5 collects the evolution with crystallization time of both, the interference function $G(s)$ and the IDF $g(r)$ for the coextruded pure PET film and the PET/PC multilayered films during isothermal crystallization at $117^{\circ} \mathrm{C}$. One sees a relative intensity increase with crystallization time due to the electron density changes within PET layers caused by the formation of crystals. Further, the shape of both $G(s)$ and $g(r)$ does not change with the thickness of PET layer, indicating that the formation of crystals within PET layers is always the same.

The first maximum of the IDF contains the information about the distribution of crystal and amorphous inter-crystalline layer thicknesses. In addition the first negative peak is related to the long period distribution. In all the films investigated, only two maxima can be observed indicating that only correlation between first neighbours occurs. This means that the stacks of crystalline lamellae consist on an average of only 3 or 4 crystallites. The variation of the long period distribution with crystallization time obtained from the IDF, first minima in all $g(r)$ of Fig. 5 , follows the same trend as the change of the long period extracted directly from the SAXS curves.

The interface distribution functions have been analyzed to obtain the parameters which describe the nanostructure of the system, i.e. distance distribution of crystalline and amorphous zones $\left(l_{1}\right.$ and $\left.l_{2}\right)$, their respective widths $\left(\sigma_{1}\right.$ and $\left.\sigma_{2}\right)$ and the distribution of long periods, $L$, where $L=l_{1}+l_{2}$ is the average long period. From the sole analysis of the IDF it is not possible to
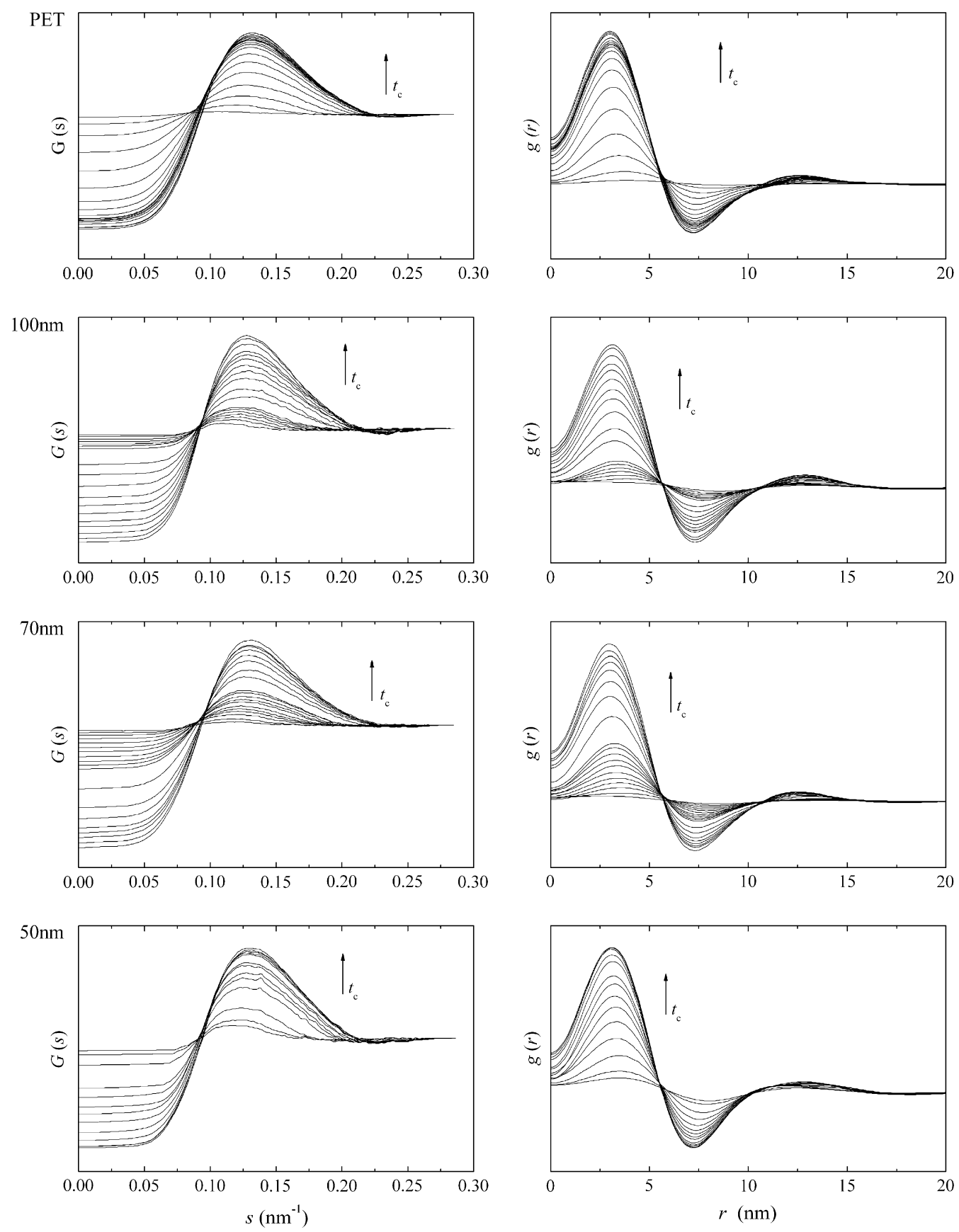

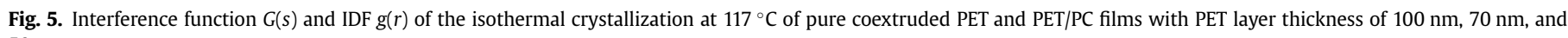
$50 \mathrm{~nm}$. 
distinguish which one of the two lengths corresponds to either the crystalline lamellae or the amorphous zones. Nevertheless it is reasonable to think that the width of distance distribution should behave as a smooth function of time. In other words, the values of $\sigma_{1}$ or $\left(\sigma_{2}\right)$ should not vary so much from the analysis of one $g(r)$ to the following one.

Fig. 6 shows the characteristic lengths $l_{1}$ and $l_{2}$ as a function of crystallization time $t_{\mathrm{c}}$ for all the films investigated. One observes that $l_{1}$ and $l_{2}$ present an opposite behaviour with crystallization
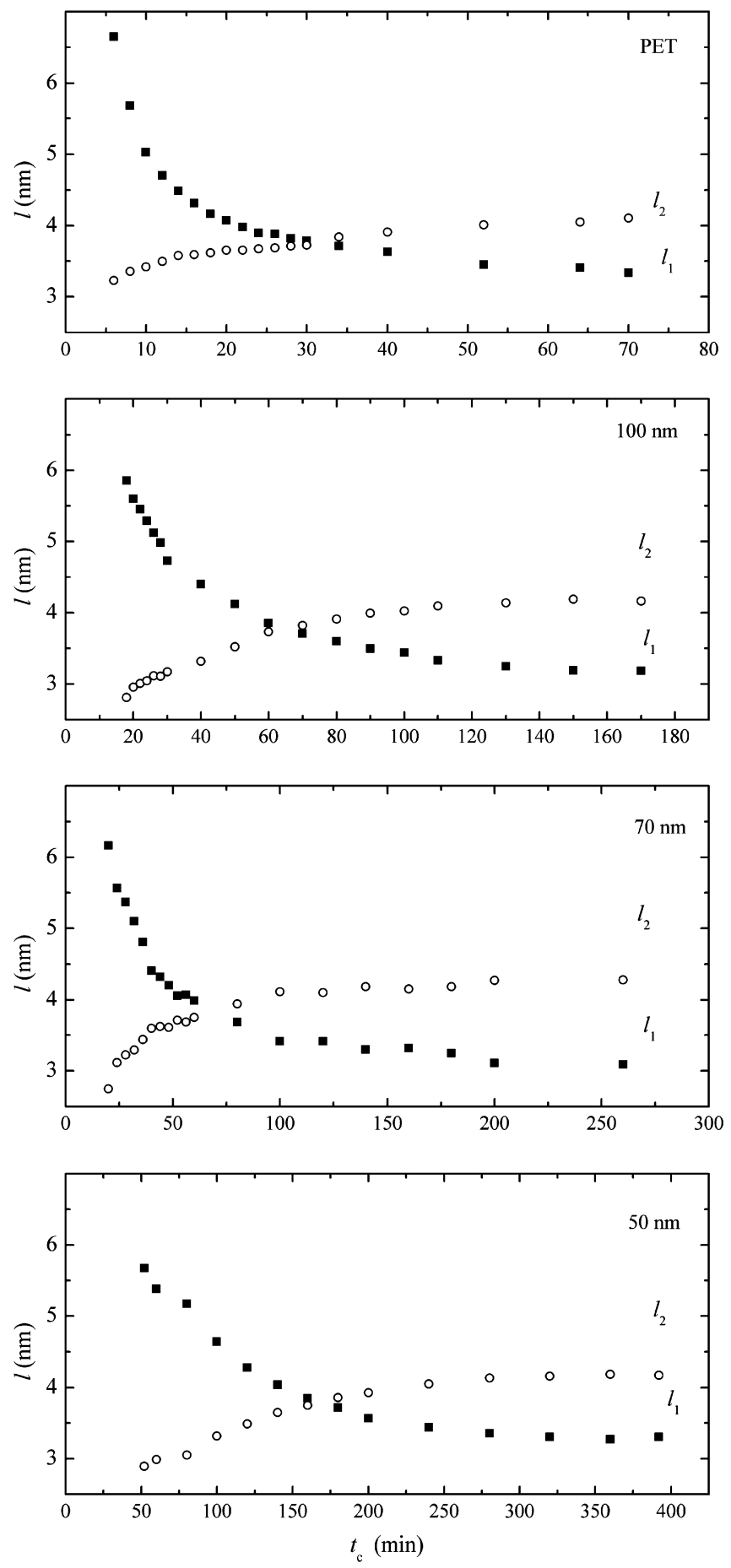

Fig. 6. Size distributions, $l_{1}$ and $l_{2}$, as a function of crystallization time, $t_{\mathrm{c}}$ for the films investigated: pure coextruded PET and PET/PC films with the thickness of $100 \mathrm{~nm}$, $70 \mathrm{~nm}$, and $50 \mathrm{~nm}$. time, $l_{1}$ decreases while $l_{2}$ increases. Considering that $l_{1}$ and $l_{2}$ represent the distribution of distances of the crystalline and amorphous zones during a crystallization process, then it is clear that the increasing function $l_{2}$ is the average distance of the crystalline zones, $l_{\mathrm{c}}$, while $l_{1}$ corresponds to the average of distance of the amorphous zones, $l_{\mathrm{a}}$.

By following the variations of $l_{\mathrm{a}}$ and $l_{\mathrm{c}}$ with crystallization time, again two different mechanisms during crystallization can be observed. First there is a period of time during which $l_{\mathrm{a}}$ decreases more rapidly than the increase of $l_{c}$. This period of time can be related to the primary crystallization, where the formation of new crystals leads to a decrease of the average correlation length of the amorphous zones $l_{\mathrm{a}}$. Subsequently, $l_{\mathrm{a}}$ and $l_{\mathrm{c}}$ present the same value and from this moment on, the decrease of $l_{\mathrm{a}}$ is the specular behaviour of the increase of $l_{c}$. This could be related to lamellar thickening, formation of new crystals or formation of new lamellar stacks (i.e. secondary crystallization). These time intervals coincide with those observed in the variation of the long period $L$ extracted from the straightforward application of Bragg's law.

When crystallization finishes, the values of $l_{\mathrm{a}}$ and $l_{\mathrm{c}}$ are very close for all samples investigated. Although it has been shown that the total degree of crystallinity decreases with increasing confinement, the average thickness of the crystals formed for all samples is of around $4 \mathrm{~nm}$ and the amorphous inter-crystalline regions of around $3 \mathrm{~nm}$. This means that the final thickness of PET crystals seems not to be influenced by the size of the PET layers. On the contrary, physical confinement of PET strongly changes the kinetics of the crystallization process. Around $60 \mathrm{~min}$ are required for PET layers of $100 \mathrm{~nm}$ to accomplish the first crystallization mechanism, while the film whose PET layers are $50 \mathrm{~nm}$ needs 175 min.

The long period variation, $L$, extracted from the analysis of the IDFs is shown in Fig. 7. As expected $L$ follows the same trend that the long period extracted from the application of Bragg's law (Fig. 3). The main difference is that lower values are found. The reason for this is that the construction of the IDFs implies that the entities which are not well correlated are removed (density fluctuations within the phases and width of phase boundaries).

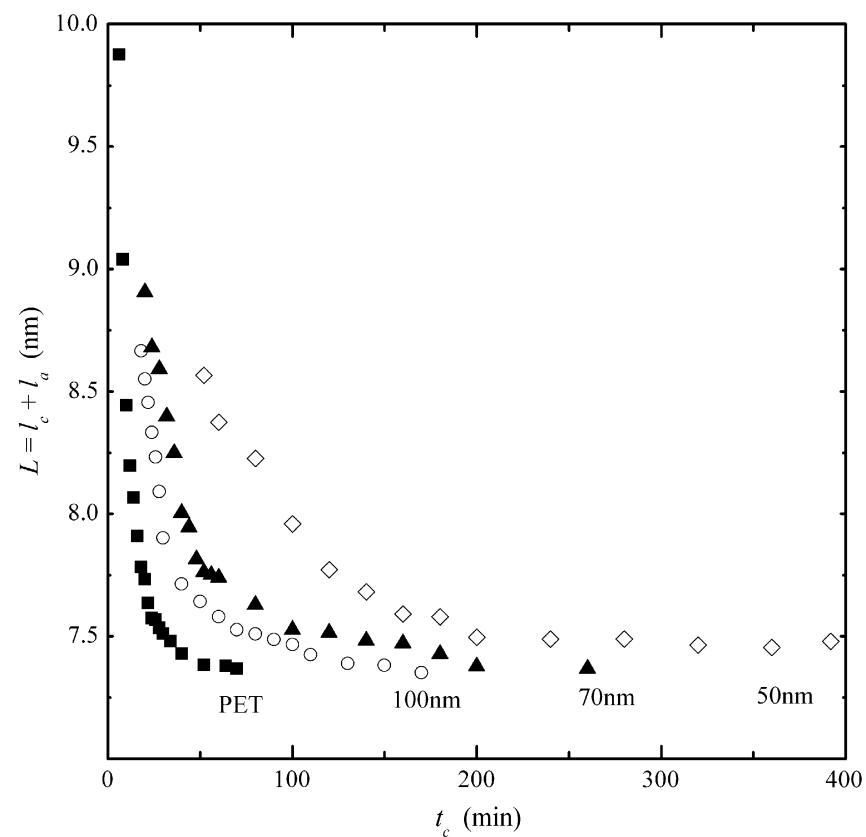

Fig. 7. Long period, $L$, from IDF analysis. 
From the values of $l_{c}, l_{\mathrm{a}}$ and $L$ is possible to derive the crystallinity of the lamellar stacks, the so called linear crystallinity, $\alpha_{\mathrm{lc}}$ $\left(\alpha_{\mathrm{lc}}=l_{\mathrm{c}} / L\right)$. Fig. 8 illustrates the evolution of the linear crystallinity and the corresponding amorphicity $\left(1-\alpha_{\mathrm{lc}}\right)$ with the crystallization time. Apart from the expected delay on crystallization process no differences are found between the linear crystallinity within the stacks of crystals formed in the coextruded pure PET films and in confined PET.
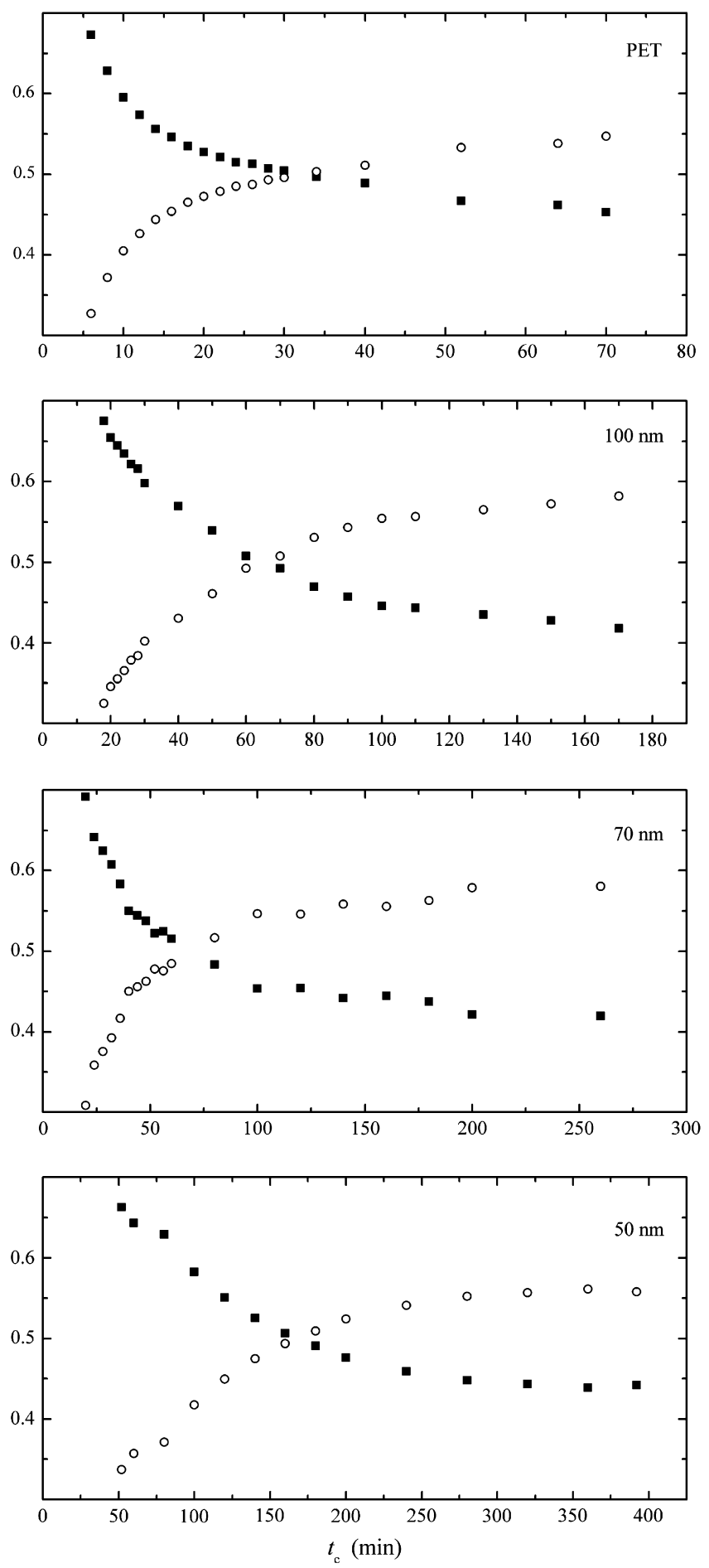

Fig. 8. Evolution of the linear crystallinity $\alpha_{\mathrm{Ic}}$ and the corresponding amorphicity, $1-\alpha_{\mathrm{lc}}$, as a function of crystallization time, $t_{\mathrm{c}}$, for the investigated films.
Finally, it is interesting to note that the value of $\alpha_{\mathrm{lc}} \sim 50 \%$, once crystallization has come to an end, is much higher than the crystallinity index values previously derived by WAXS [20]. This indicates that not all the volume of the PET layer is filled with stacks of crystals but, on the contrary, stacks of crystals are separated by large amorphous regions. The presence of stacks of crystals separated by large amorphous regions is a common feature for a semistiff polymer such a non-confined PET $[33,34]$. It has been further shown that the volume of the interstack regions rises with increasing confinement in the PET/PC films.

\section{Conclusion}

The SAXS study of the isothermal crystallization of the PET/PC multilayered films at $117^{\circ} \mathrm{C}$ as a function of the PET layer thickness has shown that the induction period and crystallization rate are influenced by the degree of physical confinement of PET. The former increases with decreasing PET layer thickness, while crystallization rate slows down with confinement.

On the contrary, the nucleation behaviour is similar for all the investigated samples and consequently it is independent of PET layer thickness.

The analysis of the SAXS curves by means of the interface distribution function (IDF) and a dual lamellar stack model shows that the lamellar stack of PET consists on an average of 3 or 4 correlated crystals. Results also reveal, as expected, that $l_{\mathrm{a}}$ decreases while $l_{c}$ increases during crystallization. No relevant differences are found among films of different PET layer thicknesses which indicates that the formed PET crystals are very similar independently of layer thickness.

Finally, the linear crystallinity (the crystallinity within lamellar stacks), is found to be much higher than the total degree of crystallinity obtained by WAXS experiments. This indicates that not all the volume of the PET is occupied with stacks of crystals but these stacks are separated by large amorphous zones in agreement with earlier findings in non-confined PET.

\section{Acknowledgment}

Financial support of this work by the Spanish Ministry of Education and Science (BFM2000-1474 and FIS2007-60534) is gratefully acknowledged. This project was supported as well by the European Community-Research Infrastructure Action under the FP6 "Structuring the European Research Area" Programme (through the Integrated Infrastructure Initiative "Integrating Activity on Synchrotron and Free Electron Laser Science"). Contract RII3-CT-2004-506008. This research was also supported by the NSF Center for Layered Polymeric Systems (grant DMR-0423914).

\section{References}

[1] Forrest JA, Dalnoki-Veress K, Durcher JR. Phys Rev E 1997;56:5705-16.

[2] Forrest JA, Mattson J. Phys Rev E 2000;61:R53-6.

[3] Fryer DS, Nealey PF, de Pablo JJ. Macromolecules 2000;33:6439-47.

[4] Torres JA, Nealey PF, de Pablo JJ. Phys Rev Lett 2000;85:3221-4.

[5] Frank B, Gast AP, Russell TP, Brown HR, Hawker C. Macromolecules 1996:6531-4.

[6] Russell TP, Kumar SK. Nature 1997;386:771-2.

[7] Fasolka MJ, Banerjee P, Mayes AM, Pickett G, Balazs AC. Macromolecules 2000;33:5702-12.

[8] Krausch G, Dai CA, Kramer EJ, Marko JF, Bates FS. Macromolecules 1993;26:5566-71.

[9] Bartczak Z, Argon AS, Cohen RE, Kowalewski T. Polymer 1999;40:2367-80.

[10] Schönherr H, Frank CW. Macromolecules 2003;36:1199-208.

[11] Reiter G, Sommer JU. J Chem Phys 2000;112:4376-83.

[12] Reiter G, Sommer JU. Phys Rev Lett 1998;80:3771-4.

[13] Destopopoulou MM, Frank CW, Miller RD, Robolt JK. Macromolecules 1996;29:5797-804.

[14] Ho RM, Chiang YW, Lin CC, Huang BH. Macromolecules 2005;38:4769-79. 
[15] Sawamura S, Miyaji H, Izumi K, Sutton SJ, Miyamoto Y. J Phys Soc Jpn 1998;63:3338-41.

[16] Jin Y, Rogunova M, Hiltner A, Baer E, Nowacki R, Galeski A, et al. J Polym Sci Part B Polym Phys 2004;42:3380-96.

[17] Schuman T, Nazarenko S, Stepanov EV, Magonov SN, Hiltner A, Baer E. Polymer 1999;40:7373-85.

[18] Baer E, Kerns, J, Hiltner A. Processing and properties of polymer microlayered systems. In: Cunha AM, Fakirov S, editors. Structure development during polymer processing. NATO science series, vol. 370E. Dordrecht: Kluwer Academic Pub. 2000; p. 327-44.

[19] Bernal-Lara TE, Liu RYF, Hiltner A, Baer E. Polymer 2005;46:3043-55.

[20] Baltá Calleja FJ, Ania F, Puente Orench I, Baer E, Hiltner A, Bernal T, et al. Prog Colloid Polym Sci 2005;130:140-8.

[21] Ruland W. Colloid Polym Sci 1977;255:417-27.

[22] Santa Cruz C, Stribeck N, Zachmann HG, Baltá Calleja FJ. Macromolecules 1991;24:5980-90.

[23] Stribeck N. X-ray scattering of soft matter. Berlin: Springer-Verlag; 2007.
[24] Zachmann HG. Nucl Instrum Methods Phys Res 1995;B97(1-4):209-15.

[25] Stribeck N, http://www.chemie.uni-hamburg.de/tmc/stribeck/dl; 2007. Downloads.

[26] Ruland W. J Appl Crystallogr 1971;4:70-3.

[27] Caceci MS, Cacheris WP. Byte 1984:340-62.

[28] Hermans JJ. Recl Trav Chim Pays-Bas 1944;63:211-8.

[29] Baert J, Van Puyvelde P. Macromol Mater Eng 2008:255-73.

[30] Kakudo M, Kasai N. In, X-ray diffraction by polymers. American Elsevier Publishing Company; 1972.

[31] Baltá Calleja FJ, Vonk CG. X-ray scattering of synthetic polymers. Amsterdam: Elsevier; 1989.

[32] Napolitano S, Wübbenhorst M. J Phys Condens Matter 2007;19:205121.

[33] Xia Z, Sue HJ, Wang Z, Avila-Orta CA, Hsiao BS. J Macromol Sci Phys $2001 ; B 40(5): 625-38$

[34] Flores A, Pierucini M, Nöchel U, Stribeck N, Baltá-Calleja FJ. Polymer 2008;49:965-73. 